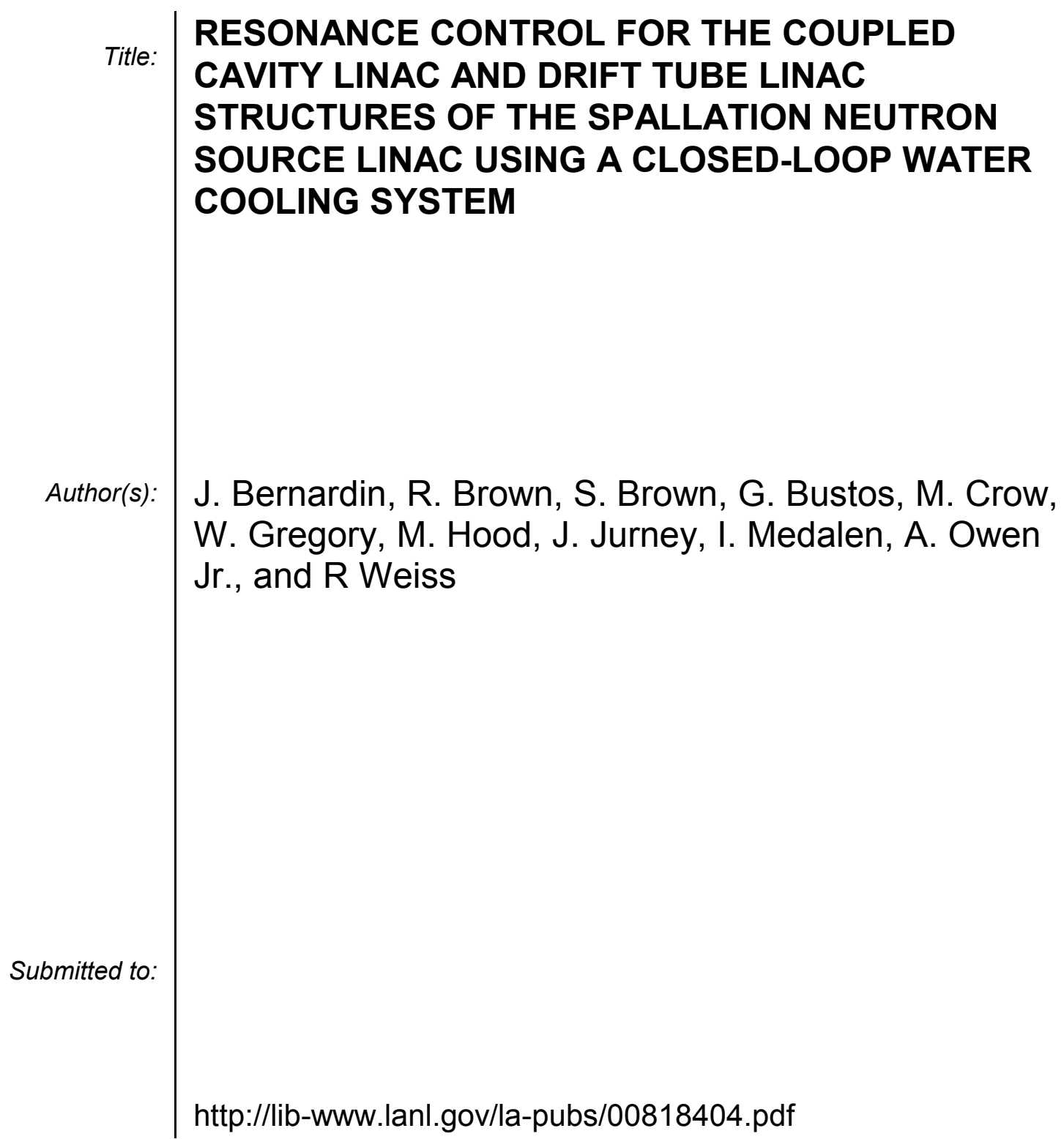




\title{
RESONANCE CONTROL FOR THE COUPLED CAVITY LINAC AND DRIFT TUBE LINAC STRUCTURES OF THE SPALLATION NEUTRON SOURCE LINAC USING A CLOSED-LOOP WATER COOLING SYSTEM
}

\author{
J. Bernardin, R. Brown, S. Brown, G. Bustos, M. Crow, W. Gregory, M. Hood, J. Jurney, I. \\ Medalen, A. Owen Jr., and R Weiss \\ Los Alamos National Laboratory, Los Alamos, NM, 87545, USA
}

\section{Abstract}

The Spallation Neutron Source (SNS) is a facility being designed for scientific and industrial research and development. SNS will generate and use neutrons as a diagnostic tool for medical purposes, material science, etc. The neutrons will be produced by bombarding a heavy metal target with a high-energy beam of protons, generated and accelerated with a linear particle accelerator, or linac. The low energy end of the linac consists of two room temperature copper structures, the drift tube linac (DTL), and the coupled cavity linac (CCL). Both of these accelerating structures use large amounts of electrical energy to accelerate the protons to an energy of $185 \mathrm{MeV}$. Approximately $60-80 \%$ of the electrical energy is dissipated in the copper structure and must be removed. This is done using specifically designed water cooling passages within the linac's copper structure. Cooling water is supplied to these cooling passages by specially designed resonance control and water cooling systems.

\section{MECHANICAL DESIGN INTRODUCTION}

The DTL is comprised of 6 large tanks each of which house a variety of accelerating components. The CCL is comprised of 4 modules, each of which contains 12 segments of 8 accelerator cavities each (total of 96 cavities per module). Models of a DTL tank and half of a CCL module are shown in Figure 1.

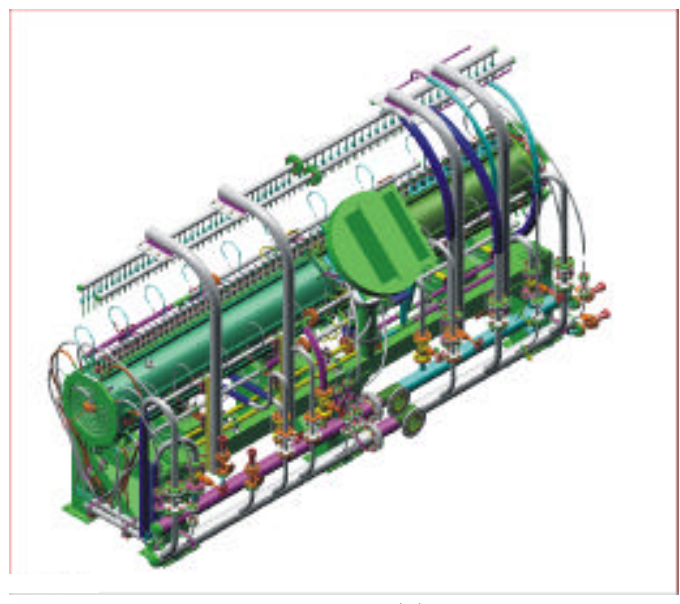

(a)

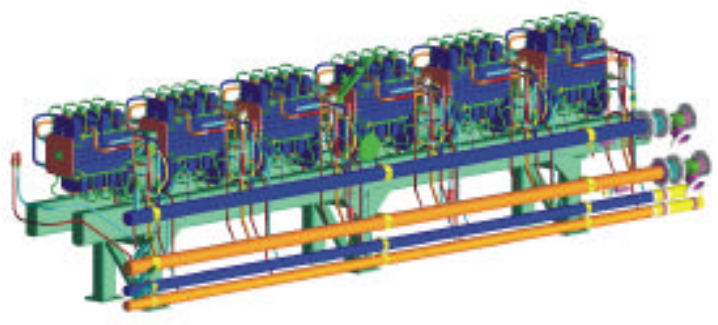

(b)

Figure 1. (a) DTL tank and (b) CCL half module.

The SNS Resonance Control Cooling System (RCCS) precisely maintains the cavity shape and resonance while controlling the thermal induced stresses. The electromagnetic field resonant frequency of a particle accelerator is a function of its internal geometry [1]. For the DTL and the CCL, the resonant frequency is mainly a function of the geometry of the tank walls (and drift tubes) and the cavities including the side coupling cells, and bridge couplers) respectively. By manipulating the dimensions of these RF structures, the resonant frequency of the particle accelerator can be finely adjusted. In practice, resonance control of a room temperature linac is maintained by both the Low Level RF (LLRF) control system and an RCCS. The goal of the SNS RCCS is to resonate the DTL at $402.500 \mathrm{MHz}$ and the CCL cavities at 805.000 MHz under nominal loaded conditions.

Rather then developing a fully integrated openloop design, modular closed-loop systems, 11 in total, will allow for much greater thermal system control along the length of the linac. Other beneficial affects are reduced start-up time, mitigation of the spread of potentially activated contaminates, consistency in design, ease of manufacturing, installation, and maintenance, and lower costs. In this closed-loop circuit, water temperature control is achieved by manipulating the hot-side (Linac side) heat exchanger water flow rate while holding the cold-side water inlet temperature and flow rate constant. 


\section{WATER SKID}

The water skid is a self-contained unit with all of the necessary plumbing, water treatment hardware, instrumentation, and pumping/heat transfer equipment required for delivering water at a desired flow rate and temperature to the RF structural components. A flow diagram is depicted in Figure 2.

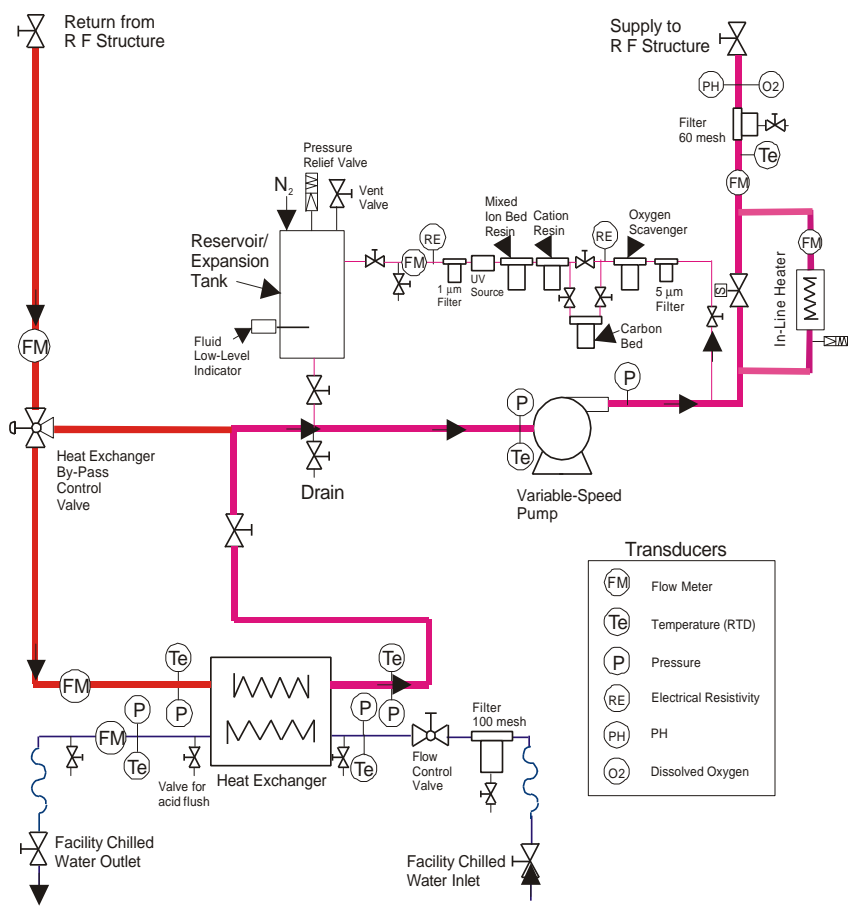

Figure 2. Water Skid Flow Diagram

The water temperature in the flow loop is manipulated by adjusting the distribution of water flow between the heat exchanger and the heat exchanger bypass line. This is achieved using a proportional 3-way valve on the return leg from the RF structure. The 3-way valve directs a portion of the water flow to the heat exchanger, and directs the remainder of the flow through the heat exchanger by-pass line. Steady-state operation requires that all of the waste heat from the RF structure be transferred to the facility's chilled water. By raising or lowering the velocity of the hot water through the heat exchanger (via the proportional by-pass valve), the overall heat transfer coefficient of the heat exchanger is raised or lowered, respectively. The net effect is that the effective thermal resistance between the water in the system and the facility's chilled water is inversely proportional to the hot water flow rate through the heat exchanger. If the chilled water source temperature and flow rate, and the thermal load of the RF structure are constant, the cooling water temperature (water temperature leaving the pump) will increase with a decrease in hot side heat exchanger water flow rate, and decrease with an increase in hot side heat exchanger water flow rate.

To remove the waste heat from the cooling loop and maintain the desired water temperature, a stainless steel flat plate counter-flowing heat exchanger was incorporated. This type of heat exchanger is relatively inexpensive to manufacture, compact, corrosion resistant and extremely efficient. The cold side of the heat exchanger is fed with chilled $7.2^{\circ} \mathrm{C}\left(45^{\circ} \mathrm{F}\right)$ water from the SNS conventional facilities. To maintain steady flow on the conventional facility side of the heat exchanger, a 2way control valve, connected to a flow meter and the PLC, was incorporated. A PLC will monitor the flow rate through the cold side of the heat exchanger and adjust the control valve to maintain the desired flow rate. To minimize contamination of the heat exchanger from the facility chilled water supply, a 100 mesh filter was added to the upstream cold side of the heat exchanger. Flushing ports will be incorporated on the cold side of the heat exchanger to allow acid cleaning to remove potential scale build-up.

A small capacity tank serves as a water reservoir and allows for expansion or contraction of the water associated with temperature changes. The tank is equipped with a Nitrogen gas source for controlling pressure and reducing the presence of dissolved oxygen in the water. A pressure relief valve, vent valve, and a liquid low-level indicator were added for safety purposes. The water reservoir feeds the main water line on the suction side of the pump through a manual valve. The reservoir tank volume has been kept to a small capacity (10 gallons) to minimize the effect of its large thermal mass on the time response of the water loop's temperature control system. A high capacity, variable speed centrifugal pump and a flow meter connected to the programmable logic controller (PLC), will be used to supply a constant water flow rate to the RF structure. Consequently, flow loop pressure fluctuations induced by the by-pass control valve will not upset the constant supply of water to the DTL.

A horizontal sealess magnetic drive centrifugal pump will be used and will have a variable speed controller. This pump is quiet and it is very unlikely that leaks will occur because it contains no mechanical seals. A flow meter will be located just downstream of the pump to monitor the flow rate and provide a feedback signal to the controller.

For greatest performance at a reduced size, a stainless steel brazed plate heat exchanger has been selected because of their efficient design, heat transfer characteristics, and history of outstanding performance.

A 3-way diverging electronically actuated control valve is located at the heat exchanger and heat exchanger bypass fluid line intersection. The valve will provide true linear proportioning, a $100 \%$ duty cycle, at least 200 incremental steps in setting position to allow for sufficient flow control resolution, and have sufficient actuation speed so as to move across its full range of motion in less than 60 seconds.

To provide for heating of the water loop (preheating of the copper structure), an inline electrical water heater was placed downstream of the pump. A solenoid valve, plumbed in parallel with the heater, will 
be used to direct all of the water flow through the heater when it is in use.

The Linac water-cooling purification system was designed with the intent of minimizing erosion, corrosion, scaling, biological growth, and hardware activation. Each component was selected to target the removal of a specific impurity, and in some cases, multiple impurities. Critical parameters to be controlled include electrical resistivity, pressure, $\mathrm{pH}$, and dissolved $\mathrm{O}_{2}$ content. Approximately 1$5 \%$ of the main water flow will be diverted to the water purification loop, the quantity being monitored with a flow meter.

The basic mechanical design of the cooling loop has helped to minimize erosion and scaling. Water flows in the cooling systems will be kept below $2.5 \mathrm{~m} / \mathrm{s}$ on surface impingement areas such as tees and elbows, and less than $5 \mathrm{~m} / \mathrm{s}$ in straight sections to reduce the effects of erosion. The narrow temperature band of the cooling water, 10 to $25^{\circ} \mathrm{C}$, reduces scaling.

A resonance control system will employ a control system that can be operated by a local, programmable logic controller, interfaced through a touchscreen interface, or it can be operated through the SNS global control system network.

\section{MANIFOLDS/TRANSFER LINES}

Connecting the water skid to the RF structure manifolds, are water supply and return lines. The transfer lines are routed from the klystron gallery, where the skid is located, to the linac tunnel through circular chases located in the ground between the buildings (approximately 20 feet in length). In the klystron gallery, the transfer lines are routed overhead, around other plumbing, cable trays, waveguides, etc. In the Linac tunnel, the transfer lines are routed along the floor between the chase exit and the RF structure manifold junctions. The transfer lines will contain isolation valves on either end for maintenance purposes. In addition, they will contain short flexible sections to aid in their installation and minimize the transmission of mechanical vibrations.

The transfer lines deliver water to a main supply manifold. From the main supply manifold, the water is diverted to a number of sub-manifolds, which in turn feed various components such as the drift tubes, post couplers, tank walls, slug tuners, dipole electro-magnets, RF window, Faraday cup, and drive iris. Proportional valves in combination with flow meters are used to accurately meter the correct amount of water to each sub-manifold.

The ultrapure deionized water, 10 to $14 \mathrm{M} \Omega$, is very aggressive and will attack materials such as copper, brass, bronze, and solders. Copper, stainless steel, brass, carbon steel, and PVC have been evaluated for use as the potential plumbing material. Copper tubing is desirable because of its mechanical properties of high strength and its ease in forming and joining, as well as being economical. Stainless steel is used in specialized water transfer systems such as those found in chemical processing facilities, microchip processing facilities, power plants, nuclear reactors, synchrotrons, and particle accelerators. Brass and mild carbon steels are susceptible to corrosion or erosion when the fluid is ultra high purity water and therefore is not recommended for use on the SNS project. Plastic and PVC materials are being avoided because each lacks strength and have a high diffusion rate for oxygen (oxygen promotes bacteria growth and enhances corrosion of copper).

The radiation emanating from a particle accelerator beam can degrade the mechanical properties of materials in close proximity to the beam centerline. This is especially true for many types of electronic components. The extent of this degradation will depend on the dose rate and cumulative radiation dose, as well as other factors such as operating temperature, mechanical stress, and exposure to air [2]. Scientists and Engineers at CERN have compiled a fairly extensive database, which relates radiation damage to cumulative dose rate for a variety of materials [3].

Copper and stainless steel, as well as many of the nonmetallic materials such as Buna-N, Hypalon, Nylon, and Neoprene meet the cumulative dose criteria of $4.3 \times 10^{6}$ Rads for SNS. However, among the nonmetallic materials, Buna-N and Neoprene have an historical usage base which indicates good results. Both have been used on the LANSCE $800 \mathrm{MeV}$ particle accelerator at Los Alamos National Laboratory with good success. The flexible Buna-N lines on the LANSCE CCL have been observed to harden over time by a combination of radiation and atmospheric damage, however they have maintained working lifetimes of well over ten years [4]. In addition, Buna-N/Neoprene hoses have been used as flexible jumper lines for the majority of the focusing and steering magnets on the LANSCE accelerator for the last twenty years [4].

Work supported by the Office of Basic Energy Science, Office of Science of the US Department of Energy, and by Oak Ridge National Laboratory.

\section{REFERENCES}

[1] Wangler, T., 1998, Principles of RF Linear Accelerators, John Wiley and Sons, Inc., NY.

[2] Sullivan, A.H., 1992, "A Guide to Radiation and Radioactivity Levels Near High Energy Particle Accelerators," Nuclear Technology Publishing, Ashford, Kent, England.

[3] Beynel, P., Meyer, P., Schonbacher, H. and Tavelet, M., 1982, "Compilation of Radiation Damage Test Data," Published as CERN Yellow Reports. Part 3, Accelerator Materials, 82-10.

[4] Boedeker, W., Meetings on LANSCE Purification Systems 6/01/99 - 8/13/99, LANSCE-2 Group, Los Alamos National Laboratory, Los Alamos, NM 